\title{
La pobreza en
}

Centroamérica: entre

agenda política,

demandas sociales y

proyectos nacionales

MSc. Jonathan Flores Martinez 
La pobreza en

Centroamérica: entre agenda política, demandas sociales y proyectos nacionales

Poverty in Central America: political agenda, social demands and national projects
MSc. Jonathan Flores Martinez Máster en Estudios de Género y Políticas de Igualdad. Catedrático de la Universidad Nacional Autónoma de Nicaragua, UNAN, Managua. https://orcid.0000-0001-8959-4682 floresjonathan57@yahoo.es. Recibido: 03 Marzo 2019 Aceptado: 15 Mayo 2019

\section{(C) (1) Copyright $\odot 2019$ UNAN-Managua}

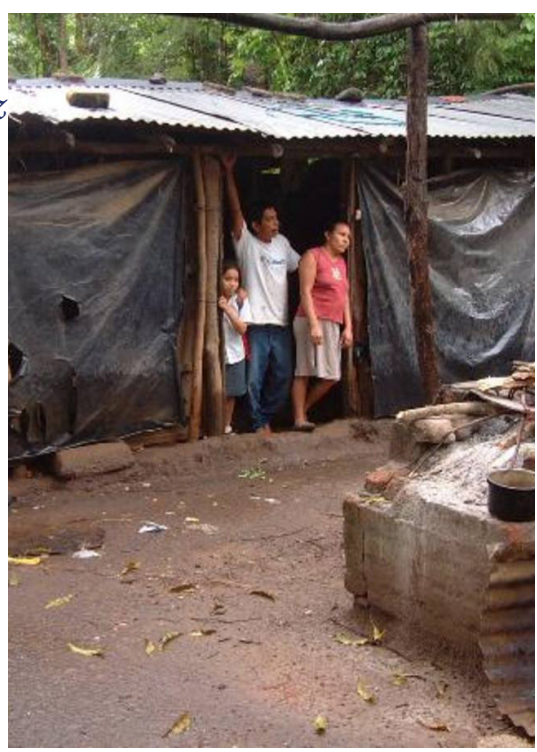

\section{RESUMEN}

Las oportunidades de desarrollo de la región centroamericana han sido condicionadas por diversos acontecimientos de carácter natural, social, cultural, y político, una región en la que su población alberga indicadores sociales deficitarios en relación a la pobreza ${ }^{1}$ y desigualdades sociales. Si bien es cierto, que la lucha contra la pobreza constituye formalmente una prioridad en las agendas gubernamentales, es relevante analizar si estas iniciativas políticas han logrado convertirse en políticas públicas y en definitiva si tienen un impacto en la mejora de las condiciones de vida para las personas de forma sostenible. Durante los últimos 15 años los países de esta región han experimentado una reducción de la pobreza, sin embargo, un alto índice de sus población continúa sumida en ella; de este panorama general se desglosa una discusión más puntual, el papel que han desempeñado los gobiernos en sus agendas programáticas, y hasta qué punto el ejercicio tradicional del poder constituye un obstáculo para enfrentar este problema. De modo que, abordar la pobreza no como un problema estrictamente econométrico, sino como fenómeno vinculado a la concepción y ejercicio del poder político es lo que orienta el interés reflexivo de este ensayo.

\section{ABSTRACT}

\section{KEY WORDS}

Poverty; political management; government; The development opportunities of the Central American region have been buman development; power; cooperation. conditioned by various events of a natural, social, cultural, and political nature, a region in which its population harbors deficit social indicators in relation to poverty and social inequalities. While it is true that the fight against poverty is formally a priority on government agendas, it is relevant to analyze whether these political initiatives have become public policies and ultimately if they have an impact on the improvement of the living conditions of people in a sustainable way. During the last 15 years, the countries of this region have experienced a reduction in poverty, however, high rates of their populations

1- Según los datos del Observatorio Centroamericano de Desarrollo Social (OCADES) (2016), señalan que más de 2.3 millones de personas salieron de la condición de pobreza en la región, sin embargo, la cantidad de personas que se encuentran en una situación vulnerable de retornar a la pobreza supera los 20 millones de personas. 
remain mired in it, a more timely discussion is broken down from this overview, the role that governments have played in its programmatic agendas, and to what extent the traditional exercise of power constitutes an obstacle to face this problem. So, addressing poverty not as a strictly econometric problem, but as a phenomenon linked to the conception and exercise of political power is what guides the reflexive interest of this essay.

\section{Introducción}

La recurrencia con que se aborda el tema de la pobreza, raras veces hace referencia a la forma de cómo se administra el poder político en una sociedad determinada, el tema generalmente, se ha enfocado más en análisis propiamente socioeconómicos, y en el mayor de los casos econométricos, tratando marginalmente sus dimensiones políticas y culturales.

Sin motivos de interferir en la lectura total de este texto, quiero prevenir al lector, que este trabajo no aborda necesariamente la medición de la pobreza en un periodo particular, mucho menos pretende ser un recetario para paliar sus efectos, ni es un análisis económico país por país.

En virtud de lo anterior, el presente artículo tiene como propósito reflexionar desde la perspectiva de la filosofía política, la relación entre pobreza y poder político. También se procura hacer un análisis sobre los rasgos característicos de la pobreza en Centroamérica y a la vez formular una crítica sobre la forma de como el poder y los medios de comunicación han instrumentalizado dicho tema, y con ello la condición y el estatus de pobre.

La perspectiva metodológica del análisis que vincula la pobreza con el poder político representa un elemento de observación clave para comprender la dinámica del desarrollo de Centroamérica y a la vez comprender el rol de los gobiernos para solucionar este problema social de dimensiones históricas y estructurales.

Estudiar la pobreza desde su arista política implica reconocer en primera instancia, que las relaciones del poder tienen sus efectos en la estructura y dinámica de cualquier sociedad. Se entiende que las demandas sociales y los recursos de carácter público no pueden ser de ningún modo manejado sin el poder político, esta condición repercute de manera directa en la vida y desarrollo de los seres humanos en cualquier circunstancia histórica.

\section{Pobreza y poder}

Teóricamente el término pobreza posee un carácter heterogéneo, sin embargo, en la actualidad perduran en el debate académico dos enfoques antagónicos que parecían superados, el primero responde a la concepción de que a cada persona le concierne la responsabilidad de gestionar su propio bienestar, es decir, que sobre el individuo recae la posibilidad de superarse 
garantizar los servicios básicos para su existencia. Desde este paradigma se asume la pobreza como un fenómeno estrictamente natural e individual (Hayek citado en Czarnecki, 2013).

Esta concepción es propia de la filosofía política neoliberal que reduce el papel interventor del Estado, como garante de los servicios públicos básicos, a desempeñar un rol de espectador frente a la realidad social, en él se presenta la política supeditada a la economía (a las directrices del mercado) como principal característica de un modelo económico desigual, regido por valores monetarios que se operacionalizan por una lógica estrictamente matemática.

Las "des-regularizaciones" propias de las politicas de inspiración neoliberal han minado la importancia de los Estados como garantes del bienestar de las poblaciones nacionales a las que se supone que cada Estado debe responder, a la vez que han favorecido la concentración de la riqueza, la desocupación, la pobreza, la desnutrición, la des-escolarización, la insalubridad, la violencia y la destrucción ambiental. (Mato, 2004: 272)

Por otro parte, la visión estructural responde a una perspectiva más compleja, asumiendo que la estructura político-económica interfiere en el estilo y calidad de vida de los individuos, lo que implica que, desde este enfoque, la pobreza está directamente asociada a la forma en cómo está organizada económicamente una sociedad y a la funcionalidad de sus instituciones políticas. (Verdara, 2007)

Esta discusión epistemológica no es reciente, data desde el siglo antepasado, Marx fue el precursor de una crítica bastante sistemática que apunta a las relaciones desiguales. Este autor, afirmaba que los privilegios de una clase social se sostenían sobre la explotación y dominio de otra mucho más vulnerable. La pobreza entendida como resultado de las contradicciones de clases y de los intereses antagónicos entre los que poseen el capital, y los que apenas cuentan con la fuerza de trabajo.

En palabras más sencillas, las instituciones sociales son las garantes de mantener el estatus quo que sostiene las relaciones sociales desiguales, este enfoque sostiene que el Estado funge como el aparato de dominación por una clase dominante que concentra la mayor parte de la riqueza y ejerce el poder sobre una amplia mayoría considerada explotada y excluida.

El tema de la pobreza no es un fenómeno reciente, existen diversos enfoques que la han estudiado desde aspectos puramente biológicos/naturales hasta lo exclusivamente social, sin embargo, la mayor parte de las investigaciones académicas e institucionales se dedican a medir, clasificar y cuantificar a los pobres, pasando por desapercibido las causas estructurales que la genera. Este paradigma rentista propugna una deshumanización de la pobreza, como categoría puramente económica o estadística.

Corona (2012) sostiene que:

En la actualidad la pobreza se estudia a partir de la definición que nos propone la economía, aun cuando hoy se presenta como una condición multifactorial, los valores que guian la elección de datos relevantes y su interpretación son resultado de consideraciones dirigidas a convertir a los pobres en sujetos productivos, si no de piedad, de compasión, o bien, que no alcanzan a satisfacer sus necesidades por incapaces. (4)

Centroamérica es una región que se caracteriza por diversos factores que influyen en sus propias dinámicas de desarrollo, en las últimas décadas, según Segovia (2007: p. 2) han sido cuatro los factores que han incidido en este cambio: los conflictos armados centroamericanos y todas sus 
consecuencias, las reformas económicas implementadas en los países ${ }^{2}$, la globalización y sus efectos, y cambios demográficos profundos.

Sin embargo, pocas veces se asocia el ejercicio del poder político con el tema de la pobreza. En Centroamérica la concepción y ejercicio oligárquico del poder fue el rasgo más notable que marcó la historia política de los incipientes Estados nacionales, autodenominados "independientes", esta tradición política heredada desde la colonia logró calar en el imaginario colectivo de las sociedades, y finalmente, se constituyó en un rasgo dominante de su cultura política, que pervive hasta el presente.

Orlando Núñez (2006) define a la oligarquía como una élite corporativa ligada al linaje familiar, a privilegios y al control administrativo de diversas instituciones, el autor también señala que la oligarquía como forma de gobierno ha estado presente en todos los países latinoamericanos y su resultado ha sido una marcada diferenciación social que tiene un sustrato ideológico heredado de las relaciones coloniales.

Este mismo autor, señala el rol que juega la ideología sobre el resto de la sociedad, afirma que: "la ideología o el proyecto dominante de las élites, será la ideología y el proyecto dominante de la sociedad, ante los cuales la población marginada debe subordinarse y someterse" (Núñez, 2006, p. 61).

La mala distribución del poder ha sido un obstáculo para lograr el desarrollo integral de las sociedades, la escasa armonía entre ciudadanía y Estado dificulta la elaboración de una agenda política que incluya las demandas sociales de los grupos más excluidos, esto a su vez, impide la elaboración de políticas públicas asociadas directamente con la realidad social de cada país e incluso de la región.

Según Foucault (1979), el poder posee dos dimensiones: una negativa y otra positiva, la primera se refiere a aquella que excluye, divide, explota, y la segunda está asociada a la producción de saberes y verdades que fomentan nuevas formas de interacción y relaciones sociales. En la región la primera dimensión es más evidente, junto al desarrollo de lo urbano pervive el atraso de lo rural, junto a la desnutrición infantil crecen las franquicias de comidas rápidas, frente al crecimiento económico permanece la desigual distribución de la riqueza. Esto implica que los pobres siguen estando excluidos de las discusiones y prioridades de las políticas públicas y su estigmatización los sitúa como parte del problema y no parte de la solución.

Por tanto, retomando la perspectiva foucaultiana, el poder es una amplia red de relaciones sociales que circula y se dirige a producir efectos a través de tácticas y estrategias específicas, es decir, se pone en práctica, se ejerce, para ello es necesario que los sujetos sean conscientes de su contexto social y de sus propias capacidades para transformar su entorno y así mismos.

El Estado figura como el principal responsable de garantizar las condiciones de una vida digna para todos los ciudadanos, sin embargo, su rol está limitado por las dinámicas del mercado y el poder transnacional, circunscribiendo en última instancia la pobreza a un problema meramente de orden económico y abordado periféricamente desde la función del mismo.

Inmersa en este orden de cosas, la gobernabilidad de la región se vuelve más compleja, puesto que el ejercicio del poder permea a todos los sujetos y grupos sociales, la inseguridad en la región no es más que el reflejo del bajo impacto de los programas gubernamentales encaminados a mejorar la calidad de vida de los ciudadanos.

2- La adopción de las políticas estándar del neoliberalismo fue una de las reformas económicas que tuvo un impacto socioeconómico deplorable para los sectores sociales más vulnerables de la región. Un programa económico que cercenó muchos derechos sociales, y el desmantelamiento de las instituciones públicas. De acuerdo con Saldomando (2006) el modelo neoliberal en Centroamérica obstaculizó las aspiraciones integracionistas de la región. 
Figuramos como una región vulnerable e inestable, los traumas de los conflictos armados en la región lentamente no han sido superados, el gasto militar representa una prioridad, la desigualdad de género y la violencia social son rasgos que predominan en las relaciones sociales, el flagelo del narcotráfico absorbe las sociedades más jóvenes y hace que los Estados orienten parte de sus recursos para frenarlo.

Este panorama no significa que no haya del todo resultados positivos en la lucha contra la pobreza y la exclusión social, sin embargo, no han tenido el impacto necesario como lo tendría si se orientará el gasto público en las prioridades sociales más sobresaliente que se deriven de un consenso entre gobernantes y gobernados.

Las relaciones desiguales de poder definen las interacciones entre ciudadanos e instituciones públicas, la participación ciudadana de igual forman está limitada por las condiciones de vida en las que se encuentran sumidas las personas, la desigualdad de poder denota a la vez desigualdad social y económica, por lo contrario, en la medida en que las desigualdades se reduzcan la cohesión social se fortalece y el desarrollo se vuelve más integral.

El discurso político y las promesas emancipadoras, han sido hasta hoy las estrategias discursivas más utilizadas para sentar una posición política acerca de la pobreza, las políticas públicas todavía no logran formularse para transformar sustancialmente los modelos de vida de los individuos. La planificación estratégica sigue siendo el principal desafío de los países de centroamericanos.

Centroamérica es un laboratorio social donde se han implementado diversos programas de "desarrollo" que redundan en cifras y prácticas discursivas, diversos modelos económicos sugeridos desde los organismos internacionales (FMI, BM) y otros impuestos desde los países más desarrollados. Estos modelos en la mayoría de los casos, sus impactos han sido superficiales, y en el peor de los casos han ampliado aún más la brecha entre ricos y pobres.

A lo anterior, se le suma que la forma en cómo la política define los problemas que afectan a la sociedad, es una condición que influye al momento de plantear soluciones. Los programas gubernamentales que se proponen para combatir la pobreza se erigen como solución directa, pero resulta que cualquier propuesta política está limitada a una definición establecida y desde un marco ideológico concreto, a su vez, esta noción termina encarnando los programas y proyectos nacionales en la práctica.

(...) el propósito de cualquier inversión pública es proponer acciones para resolver problemas o necesidades prioritarias de alguna parte de la población del pais, por ello, el análisis del problema es un aspecto fundamental para la apropiada formulación de estrategias de solución, por lo tanto, el determinar las caracteristicas de la pobreza, sus causas, aspectos históricos y contextuales, son cuestiones clave para la claridad en el estudio del problema (Buitrago, 2010:8).

Cabe destacar que las bases de la pobreza en Centroamérica no son exclusivamente materiales, sino que subyacen dimensiones simbólicas: las relaciones de poder, la concepción elitista de la política, la limitada participación ciudadana (escasa relación de la ciudanía con las instituciones públicas), son los rasgos más dominantes que se manifiestan en la vida cotidiana de los grupos sociales más excluidos. 
Por otra parte, el carácter histórico de las relaciones entre los países de la región y los países más desarrollados ha estado marcadas por realidades e intereses antagónicos que influyen en el desarrollo y superación de las disparidades socioeconómicas de sus habitantes. Las relaciones hegemónicas por parte de los más desarrollados también ejercen dominio en las relaciones exteriores de los Estados centroamericanos.

Serrano (2011) afirma que: "la confrontación Norte-Sur es la confrontación capital-trabajo, que se expresa en la concentración de capital sin precedente en el Norte y en la miseria, marginalidad y retraso tecnológico en el Sur." (p.22)

La dependencia comercial de los países centroamericanos de un único mercado (Estados Unidos) denota una débil y la vez ingénua estrategia política que condiciona su despegue en término de desarrollo económico y humano. Todavía las remesas y la cooperación externa siguen siendo los pilares económicos principales que sostienen la base económica de la mayoría de estos países.

En la región se valora como positivo el multilateralismo en todas sus dimensiones como una forma de fortalecer sus sistemas políticos y económicos. La marcada e histórica injerencia de las potencias extranjeras en la región, más en unos países que otros ha generado ciclos de retrocesos económicos, convulsiones sociales violentas y golpes de Estados como formas de control y resguardo de los intereses geopolíticos en Centroamérica.

\section{Los medios de comunicación y la pobreza.}

Los medios de comunicación solo visibilizan a los pobres de una forma negativa, amplifican la violencia social, la delincuencia, las catástrofes como fenómenos que los producen o afectan exclusivamente a ellos, se evidencia que la pobreza forma parte de la agenda mediática en la medida en que la noticia suscita la atención pública (Kitzberger \& Pérez, 2008). Los pobres como objeto de noticias hacen que los medios de comunicación en su reproducción mediática produzcan y perpetúen la estigmatización social, reproduzcan esquemas de violencia, normalizan las desigualdades, sin compromisos de ser plataformas estratégicas de transformación social.

Los medios de comunicación han estigmatizado a los pobres y han ocultado las causas reales de la pobreza. Los expertos, los organismos internacionales, los políticos oportunistas y los religiosos se han convertido en las voces oficiales para definirlos, cuantificarlos e incluso hablar en nombre de ellos.

La forma en la que los medios de comunicación abordan la pobreza revela la instrumentalización y el uso retórico de la misma, ellos son los que legitiman o deslegitiman el rol del Estado o los gobiernos; o son las plataformas donde se discuten las cifras o impacto de un programa u otro. Lo que es más evidente es que los pobres raramente figuran como sujetos con voz propia. (Kitzberger \& Pérez, 2008).

La pobreza se vuelve coyuntura social cuando las circunstancias políticas la requieren, raras veces los medios de comunicación se vuelven una plataforma para cuestionar el sistema económico desigual o la mala distribución de las riquezas. El mercado no figura como objeto de crítica, el poder extractivista de las transnacionales, se legitima por el hecho de generar empleos cuyos salarios no tienen un impacto real en la calidad de vida de los trabajadores. 
Los individuos son atiburrados a cada instante por múltiples mensajes simbólicos que lo orientan a una cultura de consumo, sin importar la condición económica o las prioridades básicas de su existencia, sino que se asume con naturalidad y resignación.

\section{La integración regional y la realidad nacional.}

Desde principios del siglo XIX, los estados centroamericanos han procurado organizarse bajo una política común, sin embargo, hasta ahora el proyecto político sigue inconcluso a pesar de compartir un patrón histórico y cultural común; todavía persisten rasgos del orden político colonial que impiden una integración sólida, y adecuada a los imperativos del nuevo contexto histórico.

La posición geopolítica de Centroamérica la convierten en una región estratégica para el comercio mundial y para los intereses de las potencias extra regionales. Ha sido definida como región proveedora de materias primas, de una densa mano de obra barata y una oportunidad de consumo provechosa para el mercado. La región representa una zona geográfica importante para el sistema económico mundial. En contraposición a esa realidad, también subsiste una parte considerable de su población que vive en condiciones precarias y exclusión socioeconómica.

La pobreza es un problema común en la región, sin embargo, no existen una propuesta conjunta para erradicarla, cada Estado-nación se empeña en resaltar sus diferencias particulares y su propio proyecto nacional. La integración real de Centroamérica figura como una idea que todavía no ha calado en el imaginario de la clase política ni de la sociedad en general. Los grupos económicos son en la práctica quienes están más interesados en la integración que los propios Estados.

Lo novedoso de la integración real que hoy está sucediendo, es que, está dirigida por el mercado y no por los Estados centroamericanos. Y por eso, tiene poco o nada que ver con lo que piensan, dicen y firman los gobiernos. No deja de resultar preocupante que esta integración se esté dando en la práctica y sin regulaciones" (Segovia, 2006).

La situación de los procesos de integración a nivel centroamericano refleja la frágil articulación que tienen los gobiernos de la región para tratar los problemas comunes. Existe una preocupación que se deriva de la fuerte influencia de los grupos económicos en los procesos políticos regionales. La concentración de la riqueza en un reducido número de personas y la débil incidencia de los actores sociales y políticos sitúan a la mayor parte de la población en una condición de marginalidad frente al poder transnacional capitalista.

El fenómeno de la globalización evidentemente no tiene el mismo impacto en los países desarrollados que en los que se encuentran en vías de desarrollo, y en estos últimos, sus Estados no han sido capaces de insertar a sus sociedades de una manera estratégica en este proceso, dado que las propias estructuras globalizadoras subyugan mediante mecanismos de dependencia, reconfiguraciones culturales y fragmentaciones políticas.

\section{Democracia y pobreza.}

El establecimiento de regímenes democráticos en Centroamérica estuvo antecedido por años de conflictos armados, tras los acuerdos de paz de los años ochenta se avizoró un panorama prometedor de bienestar y estabilidad para la región. La "democracia" entendida como la manera más viable de gobernar, supuso una alternativa para los países centroamericanos. Sin 
embargo, las democracias no han sido capaces de encontrar soluciones sostenibles al problema de la pobreza.

Los grupos sociales históricamente excluidos siguen siendo las mujeres, minorías étnicas y la población rural, la inserción de estos grupos en los procesos políticos está limitado por las desigualdades económicas y sociales en las que viven. El desarrollo de las democracias en la región está ligado a las condiciones de vida de sus sociedades.

(...) lo que la pobreza niega no es la posesión de bienes, sino la posibilidad de realizarse como seres bumanos según lo que cada uno entiende como bueno para sí, y en función de las capacidades u oportunidades que le ofrece el medio (Clemente, 2011:3)

En este sentido, la relación de la pobreza con lo político tiene efectos en lo más intrínseco de la existencia humana, como es la libertad para la realización en el sentido más amplio. La pobreza nos remite necesariamente a pensar en el sistema político y en la forma en que sus ciudadanos se involucran en los asuntos públicos.

Cuando hay pobreza en una sociedad, la participación ciudadana en los asuntos políticos se ve limitada por sus preocupaciones inmediatas, se genera indiferencia o desinterés por la política. Las condiciones sociales están relacionas a las condiciones políticas, no puede haber instituciones políticas sólidas donde hay condiciones sociales vulnerables para la vida y el bienestar de las personas.

Cabe señalar que en el plano donde convergen las condiciones socioeconómicas y políticas se corresponden al Estado como actor político por antonomasia. El rol del Estado es clave para lograr una sociedad más equitativa, en sociedades como las nuestras debe garantizar los mínimos sociales. La capacidad de los Estados de implementar políticas públicas distributivas y no acumulativas es un signo claro para constatar la clara relación que existe entre pobreza, ciudadanía y poder.

Los mecanismos políticos para que las personas canalicen sus demandas sociales y la capacidad del sistema político de satisfacerlas constituye la base estructural para comprender que lo político no no puede distanciarse de lo que aparenta ser un problema de orden meramente económico o cultural.

\section{Conclusiones.}

Se puede decir que la pobreza en Centroamérica se asocia directamente al ejercicio del poder político sin negar su multifactorialidad, las condiciones históricas han dificultado erradicarla, aún hoy en día, la mayor parte de la población de la región presenta condiciones de vida no óptimas.

Que la mala distribución del poder es un obstáculo para lograr el desarrollo integral de las sociedades, de igual manera, la escasa armonía entre ciudadanía y Estado dificulta la elaboración de una agenda política que incluya las demandas de los grupos sociales históricamente excluidos. 
De igual forma, el tema de la pobreza y la condición de los pobres constituye un elemento mediático y retórico para los medios de comunicación que despolitizan o la legitiman como condición social inmutable. Por otra parte, los rasgos predominantes de la política colonial impiden consolidar un proceso de integración política que reúnan esfuerzos inter-nacionales para resolver los problemas comunes que afectan a la región.

Por último, es imperativo destacar que estudiar la pobreza desde su dimensión política y su vínculo con la cultura política y estrictamente con el ejercicio del poder, constituye un enfoque que serviría para clarificar y comprender de una manera más integral el problema. $Y$ al mismo tiempo sugiere una oportunidad para diseñar nuevas estrategias de lucha y formulación de propuestas para su reducción a mediano y largo plazo. 


\section{Referencias bibliográficas}

- Bayón, M. (2015). La construcción del otro y el discurso de la pobreza Narrativas y experiencias desde la periferia de la ciudad de México. Revista Mexicana de Ciencias Políticas y Sociales. México.

- Buitrago, Y. (2010). El discurso de la pobreza: Análisis de los planes nacionales de desarrollo. Bogotá.

- Clemente, F. (2011). La pobreza como crítica política a la democracia: implicaciones filosóficapolíticas de la privación de capacidades básicas. Universitas Philosophica. Bogotá.

- Corona, B. (2012). la pobreza y el gobierno de los pobres, México.

- Czarnecki, L. (2013). La concepción de la pobreza en el modelo neoliberal. ¿Cómo entender la lucha contra la pobreza en México? Frontera note, 25(49), 179-191. Recuperado en 11/10/2017, de http://www.scielo.org.mx/scielo.php?script=sci_arttext\&pid=S0187$73722013000100008 \& \operatorname{lng}=$ es\&tIng=es.

- Foucault, M. (1979). La microfísica del poder. 2da Edición. La Ediciones de La Piqueta. Madrid.

- Kitzberger, P y Pérez, G. (2008). Los pobres en papel: las narrativas de la pobreza en la prensa latinoamericana. 1a ed. Konrad Adenauer Stiftung. Buenos Aires.

- Mato, D. (2004). Estado y sociedades nacionales en tiempo de neoliberalismo y globalización. En CLACSO, Consejo Latinoamericano de Ciencias Sociales (Ed). neoliberalismo; sociedad; Estado; cultura; globalización; America Latina (271-282). Buenos Aires: CLACSO.

- Muñoz, A. (s. f). Democracia y pobreza. Fundación para el Análisis y los Estudios sociales. Madrid.

- Núñez, O. (2006). La oligarquía. CIPRES. Nicaragua.

- Sáenz, R. (2007). Democracias de posguerra en Centroamérica: política, pobreza y desigualdad en Nicaragua, El Salvador y Guatemala (1979-2005). FLACSO. D.F. México.

- Saldomando, A. (2006). Aspectos económicos del neoliberalismo en Centroamérica, elementos para un debate. Recuperado en 27/07/2019, de https://cinco.org.ni/archive/16. pdf

- Segovia, A. (2007). Quien tiene el poder en Centroamérica (1): Más desiguales y cada vez más integrados. Envío, 298. Recuperado en 02/07/2019, de https://www.envio.org.ni/ articulo/3456

- Serrano, A. (2011). Obras Volumen III. América Latina ante la razón filosófica: Escritos sobre el pensamiento, la política y la cultura nicaragüense. Hispamer, CNU. Managua.

- Verdara, F. (2007). La pobreza en el Perú: un análisis de sus causas y de las políticas para enfrentarla. Lima: IEP. (Serie Análisis Económico, 24) 